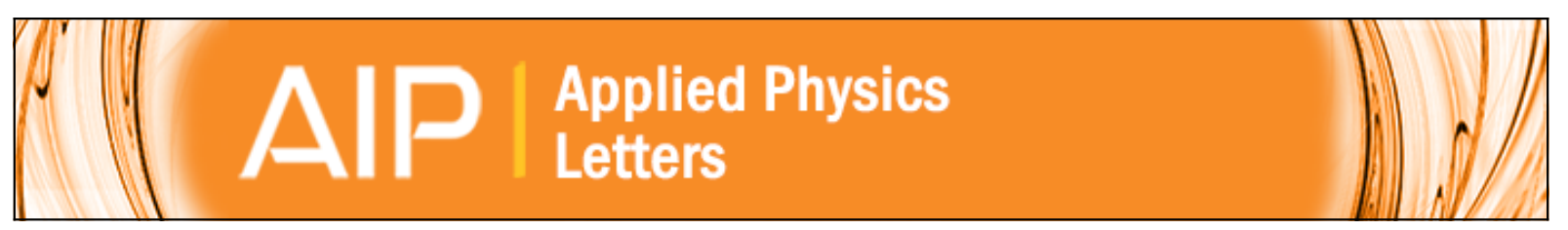

\title{
Magneto-photoluminescence of InAs/InGaAs/InAIAs quantum well structures
}

Ya. V. Terent'ev, S. N. Danilov, J. Loher, D. Schuh, D. Bougeard, D. Weiss, M. V. Durnev, S. A. Tarasenko, M.

S. Mukhin, S. V. Ivanov, and S. D. Ganichev

Citation: Applied Physics Letters 104, 101111 (2014); doi: 10.1063/1.4868644

View online: http://dx.doi.org/10.1063/1.4868644

View Table of Contents: http://scitation.aip.org/content/aip/journal/apl/104/10?ver=pdfcov

Published by the AIP Publishing

MULTIPHYSICS

SIMULATION

I. S̈FECTRUM

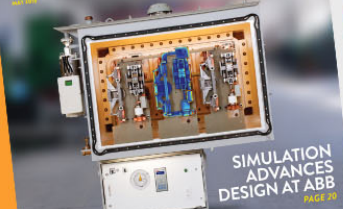

FREE Multiphysics Simulation e-Magazine 


\title{
Magneto-photoluminescence of InAs/InGaAs/InAIAs quantum well structures
}

\author{
Ya. V. Terent'ev ${ }^{1,2}$ S. N. Danilov, ${ }^{1}$ J. Loher, ${ }^{1}$ D. Schuh, ${ }^{1}$ D. Bougeard, ${ }^{1}$ D. Weiss, ${ }^{1}$ \\ M. V. Durnev, ${ }^{2}$ S. A. Tarasenko, ${ }^{2}$ M. S. Mukhin, ${ }^{2}$ S. V. Ivanov, ${ }^{2}$ and S. D. Ganichev ${ }^{1}$ \\ ${ }^{1}$ Physics Department, University of Regensburg, 93040 Regensburg, Germany \\ ${ }^{2}$ Ioffe Physical-Technical Institute, 194021 St. Petersburg, Russia
}

(Received 29 January 2014; accepted 4 March 2014; published online 14 March 2014)

\begin{abstract}
Photoluminescence (PL) and highly circularly polarized magneto-PL (up to 50\% at $6 \mathrm{~T}$ ) from two-step bandgap InAs/InGaAs/InAlAs quantum wells (QWs) are studied. Bright PL is observed up to room temperature, indicating a high quantum efficiency of the radiative recombination in these QWs. The sign of the circular polarization indicates that it stems from the spin polarization of heavy holes caused by the Zeeman effect. Although in magnetic field the PL lines are strongly circularly polarized, no energy shift between the counter-polarized PL lines was observed. The results suggest the electron and the hole $g$-factor to be of the same sign and close magnitudes. (C) 2014 AIP Publishing LLC. [http://dx.doi.org/10.1063/1.4868644]
\end{abstract}

Narrow bandgap InAs-based heterostructures, being characterized by a high carrier mobility and a strong spinorbit interaction, are generally addressed as promising systems for high-frequency electronics, optoelectronics, and spintronics. In particular, quantum wells (QWs) based on a InAs/InGaAs/InAlAs two-step bandgap engineering offer optimized confinement properties. Such QWs have been used to demonstrate a broad tunability of the emission energy in the mid-infrared range, ${ }^{1}$ high quality two-dimensional carrier systems, ${ }^{2-5}$ pronounced spin phenomena, ${ }^{6-9}$ and an electrical tunability of the electron $g$-factor. ${ }^{10}$ A precise knowledge of the carrier $g$-factors will be a key to evaluate the application potential of such systems in spintronics. Currently, reported values of electron $g$-factors determined in InAs/InGaAs/InAlAs QW by magneto-transport and terahertz experiments vary in a broad range from -3.1 to -9 depending on the In-content of the QW, ${ }^{4,6,10}$ while $g$-factors deduced from magneto-optical spectroscopy are found to be much smaller in magnitude. ${ }^{11}$ In this letter, we address the discrepancy in observed $g$-factors by reporting on polarization-resolved magnetophotoluminescence (PL) of samples with a high indium content, for which high electron $g$-factors have been reported. ${ }^{6}$ We indeed observe a strong PL polarization in a magnetic field, indicating large carrier $g$-factors and leading to a degree of circular PL polarization of up to $50 \%$ at $6 \mathrm{~T}$. We demonstrate that the spin polarization of the heavy holes determines the observed PL polarization. Interestingly, although the carrier $g$-factors are found to be high, we do not observe any energy shift between the counter-polarized PL lines, indicating in a vanishing effective electron-hole $g$-factor. From this observation, we conclude the electron and the hole $g$-factor to be of the same sign and magnitude. These findings are conserved when varying the InAs QW width.

The active region was grown by molecular beam epitaxy onto a fully relaxed $\mathrm{In}_{x} \mathrm{Al}_{1-x} \mathrm{As} /(001) \mathrm{GaAs}$ graded buffer ${ }^{12}$ with a stepwise increase of the In content $(x=0.05$ to $x=0.75)$ over $1 \mu \mathrm{m}$ and consisted of a single $\mathrm{QW}$ as sketched in the inset of Fig. 1. The QW potential barriers are built from $\operatorname{In}_{0.75} \mathrm{Al}_{0.25} \mathrm{As}$. To optimize the carrier confinement within the QW, the core of the QW, a pure InAs layer is asymmetrically embedded into a $16 \mathrm{~nm}$ thick $\mathrm{In}_{0.75} \mathrm{Ga}_{0.25}$ As. To further tailor the bandstructure and hence the $g$-factor, different widths of the InAs layer of $L_{w}=3,4$, and $6 \mathrm{~nm}$ were used. An additional structure was modulation doped with $\mathrm{Si}$ below the QW at a distance of $7.5 \mathrm{~nm}$ from the border of the QW. The doping induces a two-dimensional electron gas (2DEG) in the QW region. An electron density of $1 \times 10^{12} \mathrm{~cm}^{-2}$ and a mobility of $7 \times 10^{4} \mathrm{~cm}^{2} /(\mathrm{V} \mathrm{s})$ at $T=14 \mathrm{~K}$ was determined in magnetotransport experiments. To demonstrate the resolution of our magneto-PL setup an additional $\mathrm{In}_{x} \mathrm{Al}_{1-x} \mathrm{As} \mathrm{QW}$ structure with Mn delta-layer in the barrier has been prepared., ${ }^{7,9}$

PL detected with an FTIR spectrometer was excited by a $c w$ laser diode with wavelength $\lambda=809 \mathrm{~nm}$ and a 1-mm spot on the sample. The excitation density $W_{\text {exc }}$ was varied from 0.5 to $20 \mathrm{~W} / \mathrm{cm}^{2}$. An external magnetic field up to $6 \mathrm{~T}$ was applied perpendicularly to the wafer along the detected emission (Faraday geometry). The sample temperature was varied from 2 to $300 \mathrm{~K}$. Right- and left-handed circular polarized emission spectra were recorded applying a quarter wave ZnSe Fresnel rhomb. ${ }^{13,14}$

Bright room-temperature PL is observed from all the undoped samples, see Fig. 1. Depending on the QW width, the PL spectral position varies from $\lambda=2.4$ to $2.65 \mu \mathrm{m}$. The PL is attributed to direct optical transitions between the ground electron $e 1$ and the heavy hole $h h 1$ subbands, which is also consistent with corresponding $\boldsymbol{k} \cdot \boldsymbol{p}$ calculations. The PL spectral width is about $30 \mathrm{meV}$, being close to the thermal carrier energy. By cooling the samples down to $2 \mathrm{~K}$, the PL intensity increases by a factor of 20 and the linewidth decreases to $20 \mathrm{meV}$. In the whole range of excitation densities used, the PL intensity linearly depends on the excitation power, indicating a high quantum efficiency of radiative recombination in the QW structures.

The application of a magnetic field $B$ leads to a circular polarization of the PL, see Fig. 2(b), with a predominant emission of $\sigma^{+}$photons along the field direction. The degree of circular polarization $P_{\text {circ }}=\left(I_{+}-I_{-}\right) /\left(I_{+}+I_{-}\right)$, where $I_{+/-}$is the intensity of the $\sigma^{+/-}$circularly polarized emission is constant within the emission spectrum and exceeds $50 \%$ at $T=2 \mathrm{~K}$ and $B=6 \mathrm{~T}$. Besides, the magneto-PL lines are 


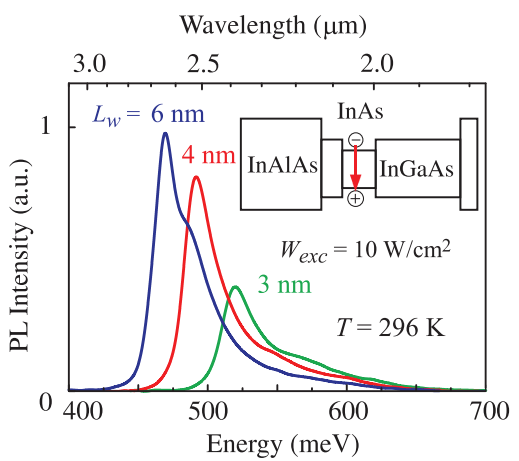

FIG. 1. PL spectra of undoped InAs QW samples. The inset shows the band diagram of the active region.

diamagnetically shifted and narrowed due to Landau quantization. Surprisingly, despite the strong circular polarization and the narrow emission lines, no energy splitting of the circularly polarized components is observed. Note that a splitting down to $0.3 \mathrm{meV}$ could be safely detected in our setup as it was observed, e.g., in a Mn-doped QWs, see inset in Fig. 2(b).

PL is also detected from the $n$-doped QW structure, Fig. 3(a). Its intensity is about one order of magnitude weaker than the one observed from the undoped QWs. Furthermore, at zero magnetic field, the PL spectrum is substantially wider and has an asymmetric shape. The linewidth corresponds approximately to the electron Fermi energy $E_{F}=60 \mathrm{meV}$. The latter value is obtained from the 2DEG density, $1 \times 10^{12} \mathrm{~cm}^{-2}$, determined by Hall measurements in the same QW structure and the effective electron mass $m_{e}^{*}=0.038 m_{0}$, determined from transport measurements in similar structures. ${ }^{2,4,6}$ The application of an external magnetic field leads to a multi-peak structure of the emission spectrum, which is caused by the formation of Landau levels, see Fig. 3(b). The data reveal that the degree of PL circular polarization is almost the same for optical transitions corresponding to the Landau level number $N=0,1,2$ and no energy shifts between the counter-polarized PL lines are

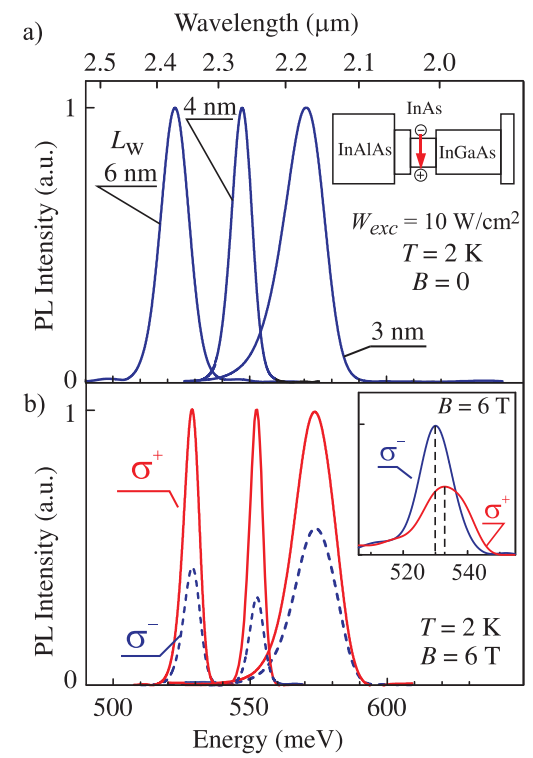

FIG. 2. (a) PL spectra of undoped InAs QWs. (b) Circularly polarized magneto-PL spectra of the same QWs. Inset shows the PL spectra from a reference structure doped with $\mathrm{Mn}$.

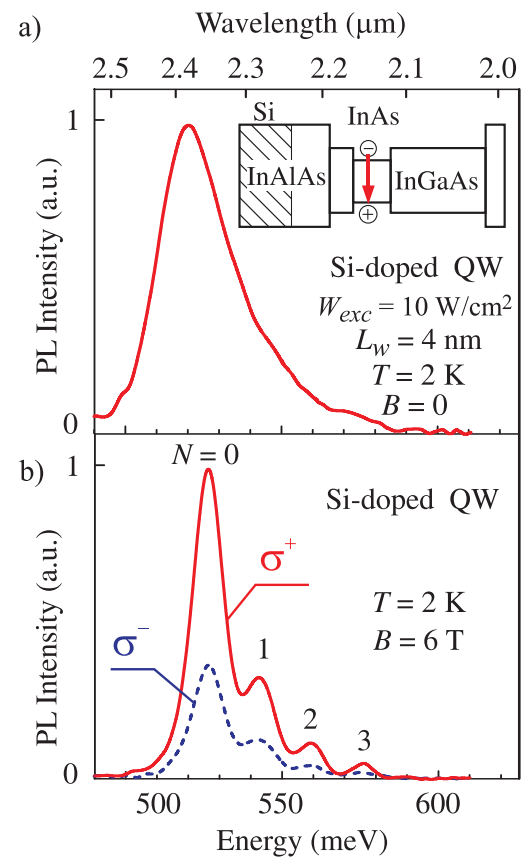

FIG. 3. (a) PL spectra of Si-doped InAs QW. (b) Circularly polarized magneto-PL spectra. The numbers $N$ denote the transitions with the corresponding Landau level numbers.

detected. The PL polarization degree reaches $50 \%$ at low temperatures and $B=6 \mathrm{~T}$ and is completely determined by the hole spin polarization since the electron spin levels below the Fermi energy are equally occupied. The energy distance between adjacent peaks is given by $e \hbar B / \mu$, where $\mu=$ $m_{e}^{*} m_{h h}^{*} /\left(m_{e}^{*}+m_{h h}^{*}\right)$ is the reduced mass, and $m_{h h}^{*}$ is the in-plane mass in the heavy-hole subband. From the data of Fig. 3, we find $\mu \approx 0.039 m_{0}$ which is close to the effective electron mass. ${ }^{2,4,6}$

The observation that $\sigma^{+}$-polarized PL dominates in both undoped and $n$-doped QWs indicates that the PL polarization results from the spin polarization of holes in the magnetic field. This is particularly clear for the doped QW, where both electron spin levels below $E_{F}$ are equally occupied. It is also true for the undoped QWs because the electron $g$-factor is known to be negative in InAs layers $\left(g_{e} \approx-15\right.$ in bulk InAs

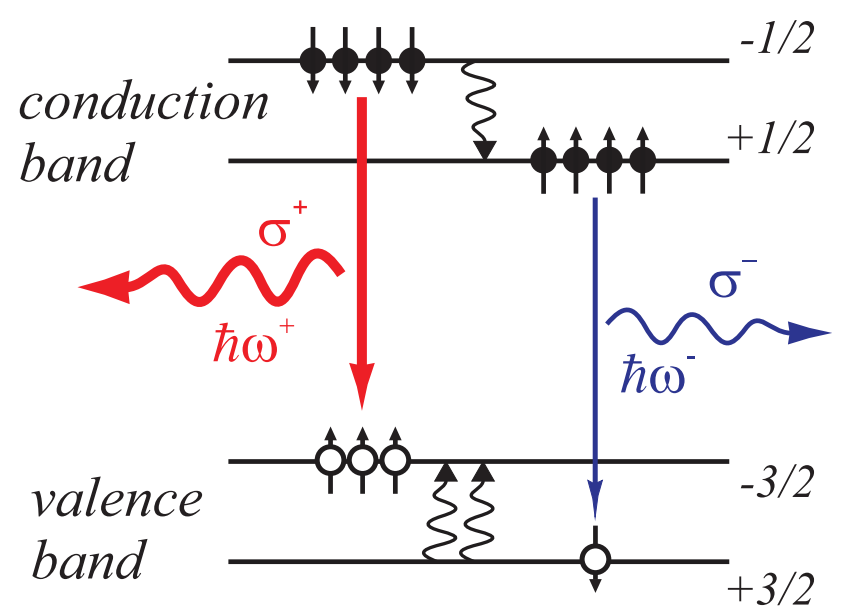

FIG. 4. Energy bands in magnetic field and allowed optical transitions. The vertical arrows indicate optical transitions with the emission of $\sigma^{+}$- and $\sigma^{-}$-polarized photons. Vertical waved arrows sketch the spin relaxation process. Electron and hole population are shown schematically by circles. 


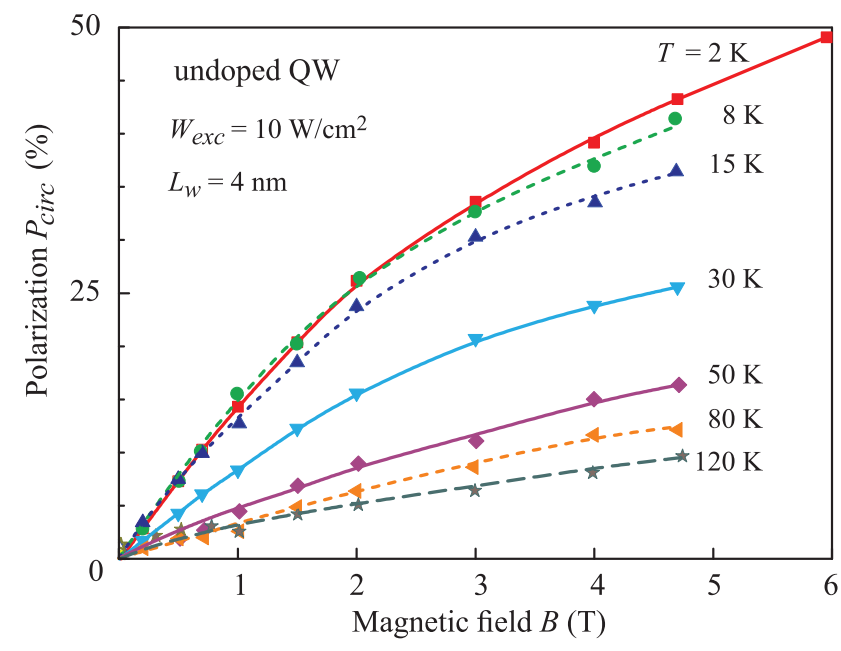

FIG. 5. Magnetic field dependencies of PL polarization measured in undoped QW. Lines are the guide for eyes.

(Ref. 15) and $g_{e} \approx-7$ as we estimate for $\operatorname{In}_{0.75} \mathrm{Ga}_{0.25} \mathrm{As}$ ). Indeed, in the case of PL polarization dominated by the electron spin polarization, the PL helicity would have to be opposite to the observed one. ${ }^{16}$ The dominating $\sigma^{+}$circular polarization of the PL also indicates that the heavy hole $g$-factor is negative and that $|h h 1,-3 / 2\rangle$ is the ground spin subband, as shown in Fig. 4 and expected from theory. ${ }^{17,18}$ The preferential population of the ground spin subband sketched in Fig. 4 is due to the fast spin relaxation of photoexcited holes. To explain the absence of a shift between the counter-polarized PL lines, we suggest that the Zeeman splittings of the electron and hole states are similar. In this case, the energies of the allowed optical transitions ${ }^{16}|e 1,+1 / 2\rangle \rightarrow$ $|h h 1,+3 / 2\rangle$ and $|e 1,-1 / 2\rangle \rightarrow|h h 1,-3 / 2\rangle$ corresponding to $\sigma^{-}$- and $\sigma^{+}$-polarized photons, respectively, are approximately the same.

Figure 5 shows the magnetic field dependence of $P_{\text {circ }}$ obtained for one of the undoped samples. The polarization increases linearly at low fields, $B \leq 2 \mathrm{~T}$ and tends to saturate at higher magnetic fields. With decreasing temperature, the polarization considerably grows, reaching 50\%. This behavior is in line with the model proposed above: $P_{\text {circ }}$ is proportional to the spin polarization of holes, which at low magnetic fields is determined by $g_{h h} \mu_{B} B /\left(k_{B} T\right)$ and tends to saturate at high $B$. Here, $g_{h h}$ is the hole $g$-factor and $\mu_{B}$ is the Bohr magneton. The degree of the electron and hole spin-polarization depends on the respective spin relaxation, which leads to different population of the spin split subbands, and recombination processes. We note that at $T<15 \mathrm{~K}$, the PL spectra are weakly sensitive to the temperature, see Fig. 5. This indicates that the carriers are efficiently heated by radiation.

To summarize, the observed bright PL in $2.5 \mu \mathrm{m}$ spectral range from InAs QW structures shows that these structures are of particular importance for optoelectronics application in the mid-infrared range. Strong circular polarization of the magneto-PL together with the absence of the line spectral splitting reveal that the $g$-factors of electrons and heavy holes while being large have the same sign and are close to each other in magnitude.

We acknowledge financial support from the DFG (SFB 689), BMBF, RFBR, RF President Grant MD-3098.2014.2, and "Dynasty" Foundation.

${ }^{1}$ E. Tournié, O. Brandt, and K. Ploog, Appl. Phys. Lett. 60, 2877 (1992).

${ }^{2}$ A. Richter, M. Koch, T. Matsuyama, Ch. Heyn, and U. Merkt, Appl. Phys. Lett. 77, 3227 (2000).

${ }^{3}$ Ch. Heyn, S. Mendach, S. Löhr, S. Beyer, S. Schnüll, and W. Hansen, J. Cryst. Growth 251, 832 (2003).

${ }^{4}$ C. H. Möller, Ch. Heyn, and D. Grundler, Appl. Phys. Lett. 83, 2181 (2003).

${ }^{5}$ M. Hirmer, D. Schuh, and W. Wegscheider, Appl. Phys. Lett. 98, 082103 (2011).

${ }^{6}$ C.-M. Hu, C. Zehnder, Ch. Heyn, and D. Heitmann, Phys. Rev. B 67, 201302(R) (2003).

${ }^{7}$ U. Wurstbauer, M. Soda, R. Jakiela, D. Schuh, D. Weiss, J. Zweck, and W. Wegscheider, J. Cryst. Growth 311, 2160 (2009).

${ }^{8}$ U. Wurstbauer, S. Sliwa, D. Weiss, T. Dietle, and W. Wegscheider, Nat. Phys. 6, 955 (2010).

${ }^{9}$ P. Olbrich, C. Zoth, P. Lutz, C. Drexler, V. V. Bel'kov, Ya. V. Terent'ev, S. A. Tarasenko, A. N. Semenov, S. V. Ivanov, D. R. Yakovlev, T. Wojtowicz, U. Wurstbauer, D. Schuh, and S. D. Ganichev, Phys. Rev. B 86, 085310 (2012).

${ }^{10}$ J. Nitta, Y. Lin, T. Akazaki, and T. Koga, Appl. Phys. Lett. 83, 4565 (2003).

${ }^{11}$ K. Tsumura, S. Nomura, T. Akazaki, and J. Nitta, Physica E 34, 315 (2006).

${ }^{12}$ F. Capotondi, G. Biasiol, D. Ercolani, V. Grillo, E. Carlino, F. Romanato, and L. Sorba, Thin Solid Films 484, 400 (2005).

${ }^{13}$ S. D. Ganichev and W. Prettl, Intense Terahertz Excitation of Semiconductors (Oxford University Press, 2006).

${ }^{14}$ E. Ziemann, S. D. Ganichev, W. Prettl, I. N. Yassievich, and V. I. Perel, J. Appl. Phys. 87, 3843 (2000).

${ }^{15}$ J. Konopka, Phys. Lett. A 26, 29 (1967).

${ }^{16}$ Optical Orientation, edited by F. Meier and B. P. Zakharchenya (Esevier Science, Amsterdam, 1984).

${ }^{17}$ R. Winkler, Spin-Orbit Coupling Effects in Two-Dimensional Electron and Hole Systems (Springer, 2003).

${ }^{18} \mathrm{M}$. V. Durnev, "Zeeman splitting of light hole in quantum wells: comparison of theory and experiments," e-print arXiv:1312.1942. 